\title{
Microglia in Alzheimer's disease
}

\author{
Heela Sarlus ${ }^{1,2}$ and Michael T. Heneka $a^{1,2,3}$ \\ ${ }^{1}$ Department of Neurodegenerative Diseases and Gerontopsychiatry, University of Bonn, Bonn, Germany. ${ }^{2}$ Deutsches Zentrum für Neurodegenerative Erkrankungen (DZNE), Bonn, Germany. ${ }^{3}$ Department of \\ Infectious Diseases and Immunology, University of Massachusetts Medical School, Worcester, Massachusetts, USA
}

\begin{abstract}
Microglia are brain-resident myeloid cells that mediate key functions to support the CNS. Microglia express a wide range of receptors that act as molecular sensors, which recognize exogenous or endogenous CNS insults and initiate an immune response. In addition to their classical immune cell function, microglia act as guardians of the brain by promoting phagocytic clearance and providing trophic support to ensure tissue repair and maintain cerebral homeostasis. Conditions associated with loss of homeostasis or tissue changes induce several dynamic microglial processes, including changes of cellular morphology, surface phenotype, secretory mediators, and proliferative responses (referred to as an "activated state"). Activated microglia represent a common pathological feature of several neurodegenerative diseases, including Alzheimer's disease (AD).

Cumulative evidence suggests that microglial inflammatory activity in AD is increased while microglial-mediated clearance mechanisms are compromised. Microglia are perpetually engaged in a mutual interaction with the surrounding environment in CNS; thus, diverse microglial reactions at different disease stages may open new avenues for therapeutic intervention and modification of inflammatory activities. In this Review, the role of microglia in the pathogenesis of AD and the modulation of microglia activity as a therapeutic modality will be discussed.
\end{abstract}

\section{Microglia}

Microglia originate from the yolk sac during primitive hematopoiesis. Their differentiation depends on the activity of the transcription factors PU.1 and interferon regulatory factor 8 (IRF8) (1-4), and their survival requires CSF1 receptor (CSF1R) signaling $(5,6)$. The brain maintains microglia levels via a finely tuned balance between local proliferation and apoptosis (7), without contributions from peripheral monocytes $(8,9)$. Microglia constitute $5 \%-12 \%$ of all glial cells in the rodent brain and $0.5 \%-16 \%$ in humans $(10-12)$. Additionally, the microglia transcriptome varies across regions, reflecting their functional diversity within the CNS (13). As the brain's resident immune cells, microglia partake in the establishment of normal neuronal connectivity and regulatory processes critical for CNS development (9), such as synaptic pruning, which ensures elimination of inappropriate synapses to strengthen the appropriate ones based on neuronal activity and experience (9). In the adult brain, microglia are involved in modulating higher cognitive functions such as learning and memory $(14,15)$. Morphologically, microglia are classified as ramified (quiescent/resting), activated, or ameboid (phagocytotic). The dynamic processes of resting microglia actively monitor the CNS parenchyma $(16,17)$, and this surveillant phenotype in combination with a specific receptor repertoire facilitates rapid microglial responses to changes in the microenvironment $(18,19)$. Further, microglia play an important role in maintaining homeostasis by removing cellular debris, dying cells, or misfolded proteins (20-25).

\section{Beyond M1 and M2 classification}

Over a decade ago, a model described the mechanism of two opposite states of macrophage activation (26). The M1 (proin-

Conflict of interest: The authors have declared that no conflict of interest exists. Reference information: J Clin Invest. 2017;127(9):3240-3249.

https://doi.org/10.1172/JCI90606. flammatory) state, characterized by production of inflammatory cytokines and nitric oxide (NO) via upregulation of inducible NO synthase 2 (iNOS/NOS2), was induced by IFN- $\gamma$ and bacterial cell wall components such as LPS or peptidoglycan. In contrast, M2 polarization in macrophages was achieved by treatment with antiinflammatory cytokines such as IL-4 and IL-13, which induced upregulation of arginase 2 (ARG2) (26). Various other signals, including IL-10, TGF- $\beta$, CSF1, and IL-6, induce diverse polarization schemes that fall under the M2 category because of their capacity to downregulate, protect, or repair in response to inflammation (27). Owing to the functional diversity of tissue macrophages, the model further expanded to M2a, M2b, and M2c classifications (28); however, recent advances in transcriptomics dispute the M1/M2 dichotomy (29), and it seems likely that macrophage activation represents a wide spectrum of very distinct responses (30).

Macrophage nomenclature was adopted to describe the different functional states of microglia (31). M1 microglia produce cytokines and chemokines (IL-1 $\beta$, IL-6, IL-12, TNF- $\alpha$, CCL2), express NADPH oxidase, and generate reactive oxygen and nitrogen species. Additionally, M1 microglia express MHC-II, integrins (CD11b, CD11c), costimulatory molecules (CD36, CD45, CD47), and $\mathrm{Fc}$ receptors. $\mathrm{M} 2$ microglia produce antiinflammatory cytokines (IL-10, TGF- $\beta$ ), growth factors (IGF-1, FGF, CSF1), and neurotrophic growth factors (nerve-derived growth factor [NGF], brain-derived neurotrophic factor [BDNF], neurotrophins, glial cell-derived neurotrophic factor [GDNF]) (32).

Despite providing valuable insights into myeloid cell complexity, transcriptome analysis of microglia derived from models of neurodegenerative diseases failed to show any clear-cut M1 or M2 signature. Instead, they found simultaneous expression of both M1 and M2 markers, suggesting the presence of mixed phenotypes or the lack of an activation signature $(13,33-35)$. Transcriptome 


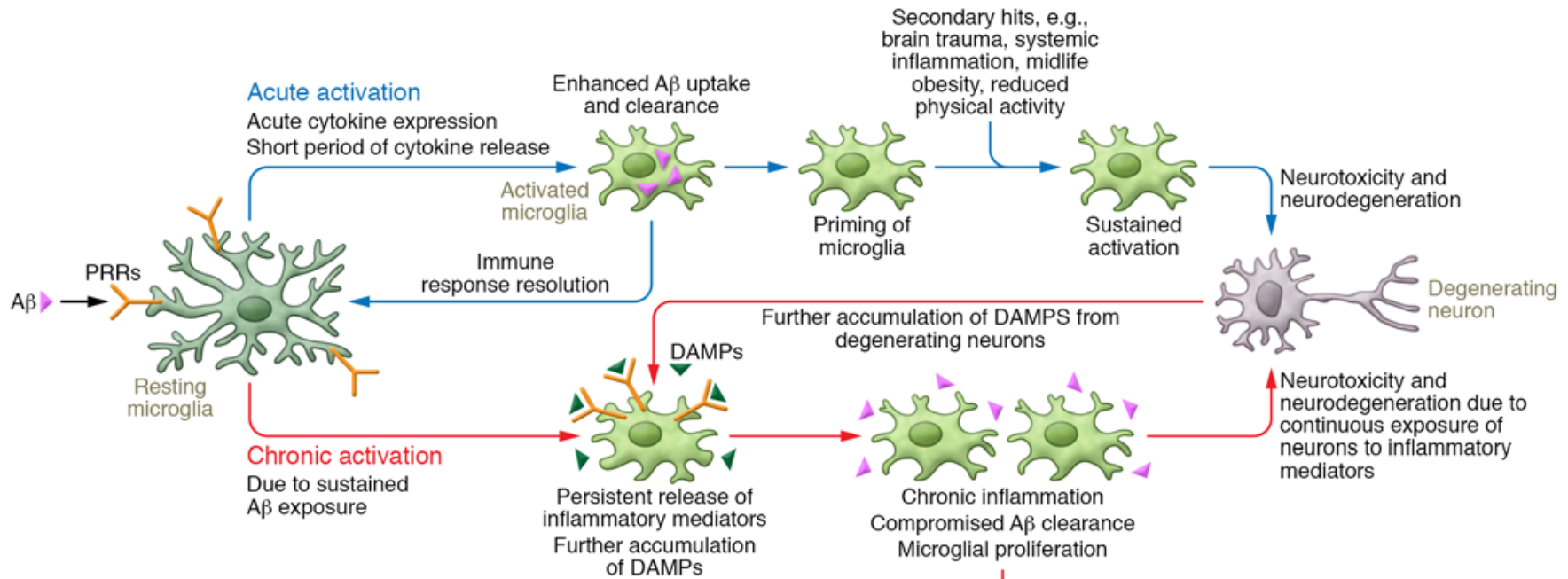
of DAMPs

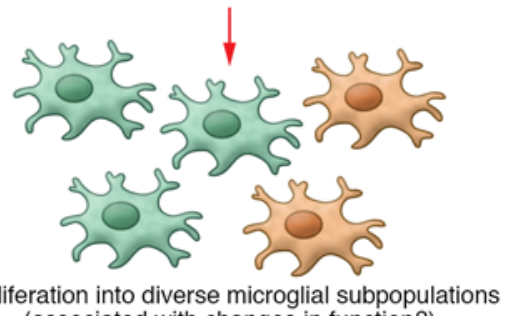

(associated with changes in function?)

Figure 1. Acute versus chronic activation of microglia in AD. A $\beta$ binds to PRRs, leading to activation of resting microglia. Acutely activated microglia express cytokines, which drive enhanced phagocytosis, uptake, and clearance of $A \beta$. Long-term activation of microglia drives proliferation as well as a chronic inflammatory state that causes neurotoxicity and neurodegeneration. Sustained activation of microglia, induced by brain trauma, systemic inflammation, obesity, and reduced physical activity, also drives neurotoxicity and neurodegeneration. DAMPs that arise from these processes further activate microglia, leading to compromised $A \beta$ phagocytosis and propagating chronic inflammation. Chronic inflammation and accumulation of $A \beta$ are well-established clinical features of $A D$.

data showed that microglia in the context of neurodegenerative diseases express both neurotoxic and neuroprotective factors as well as genes involved in stress responses, misfolded proteins, and neuronal damage. In light of these findings, M1/M2 polarization states identified in vitro are simplistic and do not model the in vivo conditions that involve continuous reciprocal interactions between microglia and surrounding CNS structures. Further, given the evidence on microglia origins and versatile functions, the M1/ M2 polarization view derived from the periphery may not translate to CNS (36). For example, the M1/M2 paradigm does not account for the role of proliferating microglia (37). Furthermore, while transcriptome studies have identified new markers selectively expressed on microglia, including the purinergic receptor P2Y12, transmembrane protein 119 (TMEM119), the chemokine receptor CX3CR1, and Siglec-H, other cell types may also display these markers $(38,39)$. Recently, Grabert and colleagues showed regional microglial heterogeneity in young mice, with the greatest convergence between cerebellar and hippocampal microglia in terms of immunoregulatoryand bioenergetics-related transcripts compared with cortex and striatum. Interestingly, aging was associated with diminished distinctions between hippocampus compared with striatum or cortex (13). The cause or impact of these changes in microglial heterogeneity in space or time remains to be further elucidated.

\section{Alzheimer's disease}

Alzheimer's disease (AD) is the most common type of dementia associated with progressive cognitive decline and memory loss.
Because aging is the primary risk factor for $\mathrm{AD}$, its prevalence is increasing with growth of the elderly population. Estimates indicate that $\mathrm{AD}$ affects more than one-third of the population over the age of 85 (40), posing an enormous socioeconomic burden. Available treatments only manage symptoms, and there is a need for therapeutics that halt or reverse the disease's underlying pathology.

$\mathrm{AD}$ is typified by distinct tissue changes associated with accumulation of extracellular amyloid- $\beta$ ( $A \beta$ ) peptides, derived from the cleavage of amyloid precursor protein (APP), and intracellular deposits of hyperphosphorylated tau. Genetically, AD is divided into familial (FAD) and sporadic forms. Fewer than about $5 \%$ of patients are affected by FAD (40) due to mutations in $A P P$, presenilin-1, or presenilin-2 (41-43), which increase either the levels or aggregation propensity of $A \beta$. Sporadic $A D$ is strongly associated with apolipoprotein $\mathrm{E} \varepsilon 4$ (APOE4) expression, whereas APOE2 expression is protective (44). Astrocytes and microglia express APOE under the control of nuclear hormone receptors, and it plays an important role in $A \beta$ phagocytosis by microglia (45), although it may influence AD through multiple mechanisms. The balance between $\mathrm{A} \beta$ production and removal seems to determine amyloid burden in $\mathrm{AD}$ brain (46), and dysregulated $A \beta$ clearance rather than increased $A \beta$ production has been linked to the etiology of sporadic AD (47). As resident phagocytes of the CNS, microglia are primarily responsible for phagocytotic clearance of $\mathrm{A} \beta$ by or local $\mathrm{A} \beta$ degradation through the release of degrading enzymes (48), although other clearance mechanisms are involved (49). 
Cumulative evidence indicates that $A \beta$ and tau aggregates are neurotoxic and trigger neurodegenerative processes in the brain, suggesting that $\mathrm{A} \beta$ and tau are central for driving $\mathrm{AD}$ pathogenesis (50-53). Interestingly, APOE4 expression was associated with enhanced $A \beta$ production, driven by increasing APP expression in neurons (54). Additionally, recent GWAS link several immune genes, particularly complement receptor-1 (CR1), CD33, and triggering receptor expressed on myeloid cells-2 (TREM2), with increased AD risk (55). Furthermore, a network-based integrative analysis of AD-related genes revealed that an immune/microglial gene network had the strongest association with $\mathrm{AD}$ neuropathology (56). These findings raise the possibility that innate immune activation may actively contribute to $\mathrm{AD}$ pathogenesis, rather than arising as a pure bystander reaction to amyloid deposition, as previously assumed (57).

\section{Microglia activation in AD}

Microglia mount an acute immune response in the brain against harmful stimuli, including misfolded proteins such as $\mathrm{A} \beta$. If the response does not resolve, the chronic activation of microglia diverts their physiological and beneficial functions (Figure 1). Activated microglia along with immunoglobulins and complement components are closely associated with $A \beta$ deposits in brains from $\mathrm{AD}$ patients and $\mathrm{AD}$ mouse models (58-62). For example, activated microglia in $\mathrm{AD}$ show increased proliferation $(63,64)$ and increased expression of inflammatory markers such as CD36, CD14, CD11c, MHC-II, and iNOS $(65,66)$, as well as markers of the M1 phenotype. However, a new phenotype of microglia, referred to as "dark microglia," was found in conditions such as chronic stress, including in the APP/PS1 mouse model of AD. Notably, dark microglia exhibited a highly activated phenotype with strong expression of CD11b and TREM2 and extensive encircling of synaptic clefts when the microglia were associated with amyloid deposits (67). This observation suggests the presence of multiple microglial phenotypes with distinct functions.

It is well known that trans-synaptic propagation of tau occurs through anatomically connected synapses. However, microglia also were implicated in spreading tau aggregates through endocytosis (via phagocytosis) and exocytosis (via exosomes) in a nonsynaptic transmission pathway (68). Depletion of microglia suppressed tau propagation (68), suggesting that microglia actively contribute to tau pathology during AD pathogenesis. Moreover, microglia may indirectly contribute to neuronal demise through regulation of neighboring astrocytes. Microglial-derived immune factors can induce a so-called A1 astrocyte phenotype that contributes to neuronal death through reduced trophic support, impaired clearance capacity, and release of toxins (69).

Microglial activation induces several dynamic changes, including morphological alterations, such as shortening of the processes and swelling of the soma, changes in surface phenotype and secretory profile, and increased proliferative responses (70). Grouping of these distinct cellular and biochemical features under the term "activation" may obscure the beneficial and detrimental outcomes of microglial responses. In AD pathogenesis, microglia activation may play a dual role: on one side, acute microglial activation in some experimental paradigms leads to decreased $\mathrm{A} \beta$ accumulation by increasing phagocytosis or clearance. In contrast, chronic activation of microglia contributes to neurotoxicity and synapse loss by triggering several proinflammatory cascades. Microglia express pattern recognition receptors (PRRs), an evolutionarily conserved family of innate immune cell receptors that are designed to respond to danger- or pathogen-associated molecular patterns (DAMPs or PAMPs). PRRs bind to different species of $A \beta$ with various affinities (71). A recent study showed that under certain circumstances in human $\mathrm{AD}$ cortex, highmolecular-weight $A \beta$ species dissociate into small neurotoxic oligomeric $A \beta$ species, and administration of the latter to mice activates microglia in vivo. These activated microglia were characterized by increased microglial CD68 levels and decreased ramification (72). In vitro, $A \beta$ activates microglia by binding to PRRs, including receptors for advanced glycation end products (RAGE) (73), TLRs (74), and scavenger receptors $(75,76)$. Importantly, upon PAMP or DAMP binding to PRRs, the enhanced production of proinflammatory cytokines such as IL- $1 \beta$ and TNF- $\alpha$ and other inflammatory mediators compromises microglial phagocytosis $(77,78)$. Interestingly, the microglia cytokine expression profile seems to influence the microglia phagocytotic index. Microglia producing IL- $1 \alpha$ and IL-1R $\alpha$ showed a higher phagocytic index and $A \beta$ load than microglia that did not produce these cytokines. Interestingly, TNF- $\alpha$ - and IL- $1 \beta$-producing microglia were abundant in $\mathrm{APP} / \mathrm{PS} 1 \mathrm{dE} 9 \mathrm{AD}$ model mice and were associated with lower $\mathrm{A} \beta$ load and phagocytic index (79). However, most experimental data concerning microglial $A \beta$ phagocytosis published to date must be considered with great care, as the assays used measured mostly uptake but not intracellular A $\beta$ degradation. Importantly, during neurodegeneration, additional DAMPs may arise, including but not restricted to chromogranin A (80) or myloid-related protein 14 (MRP14) (81), which by themselves can activate microglia and compromise phagocytic clearance. It is likely that such factors, together with accumulating $A \beta$, are responsible for the chronic neuroinflammation observed in AD.

\section{Loss of inhibitory signals}

Under physiological conditions, microglia retain a neural-specific phenotype (82) and remain in a relatively quiescent state due to mutual exchange of neuronal and astrocytic signals $(83,84)$. Neuronmicroglial inhibitory interactions are facilitated by binding of a variety of neuronal immunomodulators to their cognate receptors expressed predominantly on microglia, such as CD200/CD200R (85, 86), CX3CL1 (fractalkine)/CX3CLR1 (87, 88), CD22/CD45 (89), CD172A/CD47 (90), CD95/APO-1, and ICAM5/lymphocyte function-associated antigen-1 (LFA1) (91). Dysregulated neuronmicroglia communication has been implicated in neurodegenerative diseases (92). Studies using murine models of $\mathrm{AD}$ have demonstrated that chemokine receptors influence AD pathology by modulating several aspects of microglial function, such as accumulation, clustering, and phagocytosis (93-96). Loss of the chemokine receptor CXCR3 in APP/PS1 mice reduced A $\beta$ burden and inflammation while increasing BDNF expression and improving behavioral deficits (95). Thus, CXCR3 signaling is involved in the suppression of microglial $\mathrm{A} \beta$ phagocytosis in APP/PS1 mice, ultimately leading to an increase of $A \beta$ plaque deposition. In contrast, CX3CL1 or CX3CR1 deficiency promoted microglial phagocytosis of $A \beta(96,97)$. However, CX3CL1 deletion increased tau phosphorylation (97), sug- 
gesting that the divergent effects of CX3CL1 signaling in AD pathology should be considered for pharmacological targeting of neuroimmunomodulators. In addition to surface receptor interaction, neurons may also modulate microglial function and structure through the release of neurotransmitters. For example, tyrosine hydroxylase ${ }^{+}$ $\left(\mathrm{TH}^{+}\right)$neurons in the locus coeruleus (LC), a small brainstem nucleus, regulate microglial mediator release and $\mathrm{A} \beta$ clearance in their projection areas, including the hippocampus and neocortex, through the release of norepinephrine (98). In turn, early LC degeneration and subsequent loss of norepinephrine in the respective brain regions permit increased immune responses and $A \beta$ accumulation (99).

\section{Multifaceted immune activation}

In vivo imaging shows that microglia activation precedes the onset of $\mathrm{AD}(100)$ and is paralleled by increased levels of inflammatory markers in the cerebrospinal fluid (101), supporting the idea that inflammation occurs early in $\mathrm{AD}$ pathogenesis. In patients with mild cognitive impairment (MCI), a prodromal stage of $A D$, reductions in microglial activation were observed after a 2-year follow-up. In contrast, $\mathrm{AD}$ patients showed an increase in microglial activation (102). Interestingly, in MCI, microglial activation was positively correlated with cognition and gray matter volume, suggesting that microglial activation plays a protective role in the early phase of clinically apparent disease (100). In contrast, microglial activation correlated with glucose hypometabolism in AD patients (103), suggesting a link between microglial activation and synaptic dysfunction. Taken together, the authors suggested a bimodal distribution of microglial activation in the trajectory of $\mathrm{AD}$, with an early protective peak at the preclinical/prodromal stage and a later cidal proinflammatory phenotype peaking at the clinical stage (102).

This hypothesis is supported by the findings of the Alzheimer's Disease Anti-inflammatory Prevention Trial (ADAPT), in which patients were stratified into three phases depending on their level of cognitive decline. This stratification reasoned that patients with a fast decline in cognition would probably be closer to clinically apparent $\mathrm{AD}$ than those with little or no cognitive decline (104). Furthermore, epidemiological studies supported that NSAIDs decrease the development of AD (105). Their main targets were cyclooxygenase enzymes: constitutively expressed COX-1 and inflammation-induced COX-2. Interestingly, the COX-1 inhibitor naproxen was beneficial in fast decliners only; in contrast, the selective COX-2 inhibitor celecoxib was beneficial in slow decliners but harmful in the fast-progressing group. COX-2 expression peaks in neurons in Braak phases O-II (an early stage characterized by neurofibrillary changes beginning at transentorhinal regions), probably representing an adaptive response that subsequently declines (105). Thus, COX-2 blockade in early phases may be harmful, whereas COX-1 inhibition (in addition to COX-2 inhibition) may be beneficial, as COX-1 expression in microglia increases during Braak phases III-IV (when the neurofibrillary tangles spread to the hippocampus) (105), although this does not exclude the possibility of other mechanisms. However, in the clinical phase of AD, the strong association between microglial activation and $\mathrm{AD}$ pathology was observed in young $\mathrm{AD}$ patients, but activation waned in AD patients older than 80 years (106), suggesting that the immune activation state in the trajectory of $\mathrm{AD}$ might suffer a subsequent decline. Thus, a detailed longitudinal characterization of microglia activation in relation to AD pathogenesis is needed. This will be important for determining the therapeutic outcome of immunotherapy.

\section{Toll-like receptors}

TLR4, TLR1, TLR2, TLR9, their coreceptors CD14 and CD36, and NOD-like receptors such as the NLRP3 inflammasome are among the well-characterized PRRs in AD subjects and related murine models of AD. TLRs bind both fibrillar and oligomeric forms of A $\beta$ (74). Increased expression of Tlr2, Tlr4, Tlr5, Tlr7, and Tlr9 mRNAs was found in $A \beta$ plaques, and expression of $T l r 2 / 5$ was elevated in plaque-associated tissue in comparison with plaquefree tissue (107), suggesting a topographic TLR distribution in association with AD pathology. Stewart and colleagues demonstrated that $A \beta$-mediated release of proinflammatory factors and neurotoxicity partially involved signaling through the CD36/ TLR4/TRL6 complex (108). Importantly, studies elucidating the role of TLRs in microglial activation have yielded conflicting results (109). APP/PS1dE9 mice lacking functional TRL4 showed an increase in $A \beta$ load associated with decreased expression of IL-1 $\beta$, CCL3, and CCL4 and decreased cognitive function (110), suggesting a protective role of TRL4 in amyloid clearance. Similarly, a single intrahippocampal injection of LPS, a well-defined TLR4 agonist, boosted amyloid clearance in APP mice. This clearance was associated with a transient increase in proinflammatory mediators (111), which may be positively involved in this clearance reaction. In contrast, studies of acute (109) and chronic LPS exposure reported accelerated amyloidosis and increased microglial activation that localized to plaques in LPS-treated brains (112, 113). Chronic LPS challenge could potentially induce tolerance, which may shift the microglial state from promoting clearance to accelerating deposition.

Mice lacking the TLR2/4 coreceptor CD14 exhibited a reduction in amyloidosis and CD45 and ionized calcium-binding adapter-1 (IBA1) immunoreactivity. Interestingly, this was associated with an increase in M1 markers such as TNF- $\alpha$ and IFN- $\gamma$, a decrease in M2 markers including Fizz1 and YM1, and, paradoxically, an increase in the M2-associated marker IL-10 (114). These findings suggested that CD14 deletion shifts microglia activation to an immunomodulatory state, possibly by blocking TLR2 signaling, which contributes to inflammation and AD pathology (115). A drawback of TLR4 stimulation was the observed increase in taurelated pathology in 3xTgAD and rTg4510 mice (109). Based on these findings, Scholtzova and colleagues tested TLR9 stimulation with CpG-containing oligodeoxynucleotides (CpG-ODNs) in mice, demonstrating that repeated monthly injections of $\mathrm{CpG}-\mathrm{ODNs}$ reduced amyloid deposition and cerebral amyloid angiopathy, and prevented cognitive deficits. In contrast to TLR4 stimulation, TLR9 stimulation decreased tau-related pathology (116). With regard to microglial activation, overall levels of CD45, CD206, and CD11b were reduced in mice treated with CpG-ODNs, although microglia within the few remaining amyloid deposits showed increased CD45 reactivity (117). Plasma levels of both pro- and antiinflammatory cytokines were elevated; importantly, IL-1 $\beta$, previously associated with increased tau pathology, remained unchanged (117). Likewise, a single i.c.v. injection of CpG-ODNs 
decreased the cerebral $A \beta$ load and rescued deficits in fear conditioning (118). Another interesting comparison of TLR4 and TLR9 manipulation is the transient nature of the TLR9-associated inflammatory response. Longitudinal assessment of TLR4 stimulation revealed a transient increase in proinflammatory markers such as IL-1 $\beta$ and TNF- $\alpha$, and in microglia it transiently increased TLR4, CD45, CR3, scavenger receptor A (SR-A/CD204), and Fc $\gamma$ receptor immunoreactivity in the brain (111). Although the levels of all markers analyzed returned to baseline within a week, microglial CD45 and CR3 levels remained elevated at 28 days after injection. In contrast, 12 hours after TLR9 stimulation, CD45, CD11b, and CD206 levels remained elevated, but there was an overall reduction in these markers with the longitudinal stimulation regimen (117). These observations indicate that a transient surge in proinflammatory mediators that eventually subsides might be beneficial in promoting clearance without driving excessive inflammation. Interestingly, a polymorphism in the TLR9 promoter was associated with decreased AD risk, and further analysis of peripheral blood monocytes suggested that the polymorphism was associated with increased TLR9 expression (119). Hence, it seems that TLR9 stimulation activates an innate immune response that benefits $\mathrm{AD}$ pathology without provoking inflammation.

\section{Complement system}

Given the role of microglia and complement in synaptic pruning and the identification of a CR1 gene variant that is linked to an increased risk for $\operatorname{AD}(17,120)$, complement-mediated microglial phagocytosis of synapses may contribute to $\mathrm{AD}$ pathogenesis. Accordingly, Hong and colleagues demonstrated that microglia in concert with complement caused early synapse loss when challenged with oligomeric $A \beta$ (121). Before the onset of $A \beta$ deposition, microglia engulf synapses opsonized with the complement factors $\mathrm{C} 1 \mathrm{q}$ and $\mathrm{C} 3$ upon CR3-mediated recognition of C3 (121). Blocking of C3, C1q, or CR3 rescued this early synapse loss in APP/PS1 mice (121). However, other studies have reported that inhibition of different components of complement impaired $A \beta$ phagocytosis, resulting in elevated synapse loss. In a mouse model of $\mathrm{AD}$, overexpression of soluble complement component $3 \mathrm{~b} / 4 \mathrm{~b}$ receptor 1-like (CRRY; a complement inhibitor in rodents) (122), C3 deficiency (123), or blocking of C5a (124), a downstream component of the complement cascade, increased $A \beta$ levels in the absence of increased $A \beta$ production, reflecting the role of complement in microglial phagocytosis. In the same studies, complement blockade was associated with increased neurodegeneration. In contrast to these observations, Lian and colleagues demonstrated that $\mathrm{A} \beta$ induced release of $\mathrm{C} 3$ from astrocytes and impaired microglial A $\beta$ phagocytosis (125). Inhibition of microglial $\mathrm{C} 3 \mathrm{aR}$ reduced microglial activation and augmented $\mathrm{A} \beta$ clearance in the brain (125), suggesting a detrimental role for complement. Interestingly, the Hong et al. study associated the C3 complement cascade with increased microglial phagocytosis of synapses in an early phase of $\mathrm{AD}$, prior to $\mathrm{A} \beta$ deposits (121), whereas Lian and colleagues demonstrated C3-mediated impairment of microglial phagocytosis during robust $A \beta$ pathology (125). In a report from Maier and colleagues, C3 deficiency was detrimental only during the late phase of $\mathrm{AD}$ in 17-month-old mice (123). The discrepancies among these studies underscore the impact of the precise disease time period on complement-mediated regulation of microglial phagocytosis. Chronicity could be another factor that influences the role of complement in AD. For example, acute C3 exposure increased microglial phagocytosis in vitro, while chronic exposure diminished microglial phagocytosis (125), suggesting that chronic $\mathrm{A} \beta$ load may divert initial phagocytosis-promoting mechanisms to mechanisms that promote deposition. Thus, complement's role in $\mathrm{AD}$ pathogenesis needs to be examined further, taking into account disease phase and the particular targets being considered for therapeutic intervention.

\section{Cytokines}

Another approach to manipulate microglial responses to $A \beta$ was to regulate cytokine levels. IL-1 $\beta$, a key cytokine of the innate immune response, was shown to enhance A $\beta$ pathology (126), as well as increase tau pathology (109). Moreover, mice overexpressing IL-6 (127) or IFN- $\gamma$ (128) had enhanced microglial phagocytosis of $A \beta$ accompanied by an increase in complement component mRNA levels, TNF- $\alpha$, and other inflammatory mediators. TNF receptor (TNFR) deficiency in 3xTgAD mice was associated with increased amyloid deposition and enhanced tau phosphorylation (129). Moreover, microglia from TNFR-deficient 3xTgAD mice showed defective phagocytosis, highlighting the protective role of TNF- $\alpha$ in facilitating clearance mechanisms. These studies suggest that a perpetual proinflammatory environment in the context of $A \beta$ deposition may have beneficial outcomes. However, the inhibition of proinflammatory response, including loss of NLRP3 inflammasome, myeloid-related protein-14 (MRP14), and IL-12 and IL-23 signaling, has also been beneficial in reducing amyloid pathology (130). The NLRP3 inflammasome is critical for maturation of IL-1 $\beta$, and loss of NLRP3 in APP/PS1 decreased amyloid burden (131).

The innate immune system can be harnessed to clear $A \beta$ in the context of antiinflammatory cytokines. IL-10 levels are elevated in $\mathrm{AD}$ brain, and IL-10 overexpression in mice increased APOE expression in addition to worsening AD-related pathology and cognitive impairment (132). Il10 deletion led to increased $\mathrm{A} \beta$ phagocytosis and increased CD68 reactivity in A $\beta$ depositassociated microglia (133). Thus, cytokines could serve as potential therapeutic targets to modulate microglial responses. Antibodies targeting proinflammatory cytokines such as IL-1, IL-6, or their receptors are used in the treatment of autoimmune diseases and may have beneficial effects in $\mathrm{AD}$; however, the precise window of opportunity and ideal cytokine target still need to be identified.

\section{Microglia proliferation}

Microglial activation during injury involves recruitment of cells, mainly through local proliferation of resident microglia, followed by a wave of apoptosis to reduce the levels to baseline (134). Little is known about microglial proliferative responses in the brain. Increased microglial proliferation has been described in the brains of $\mathrm{AD}$ patients (135), and in the prodromal stage of Parkinson disease (136), suggesting that microglial proliferation is an early event in the course of neurodegenerative diseases. Increased microglial proliferation was observed in several murine AD models (63), appearing with plaque onset and persisting for 18 months (63). In mice, approximately $50 \%$ of the proliferating microglia were found near $A \beta$ deposits (63), whereas in human $A D$, the majority of plaque-associated microglia were not proliferating 
(137). In focal cerebral ischemia, proliferating microglia mediate neuroprotection via secretion of growth factors (138). Microglial proliferation in the brain peaked between 48 and 72 hours after injury (139), and selective ablation of proliferating microglia was associated with increased ischemic lesion size and neurodegeneration (138). In the absence of pathology, microglia proliferation increased in the cortex of mice subjected to environmental enrichment and wheel running, and the proliferating microglia appeared ramified, without signs of activation (140).

Lawson and colleagues reported a microglial turnover rate of $0.05 \%$ at any given time in healthy brain (141), but recent data from the same group indicated a turnover rate of $0.69 \%$ (7). These data estimate that it takes approximately 96 days to renew the entire microglial population in mice, suggesting several cycles of microglial self-renewal during a mouse's lifetime. The number of self-renewal cycles in the human brain was estimated to be around 100 (7). This striking difference between human and mouse supports the idea that rodent microglia may not experience pathological factors associated with aging such as senescence or dystrophy (70). These findings raise the question of how many cell divisions can occur before microglia suffer from critical shortening of telomeres and enter a senescent, dystrophic state. How do increased self-renewal cycles due to pathology influence the physiological functions of microglia? The role of microglia proliferation in relation to $\mathrm{AD}$ needs further examination.

Microglia receive signals for survival and proliferation following stimulation of the CSF1 receptor (CSF1R) with CSF1 or IL-34 (142). At the transcriptional level, the CEBP/ $\alpha /$ PU.1 pathway is involved in the regulation of monopoiesis and granulopoiesis $(143,144)$ and serves as a candidate for regulating proliferation in microglia. Elevated expression of CSF1R pathway components were found in the brains of $\mathrm{AD}$ patients. Furthermore, the expression of several proliferation and inflammation markers correlated with disease severity (64), suggesting a pathological role for microglia proliferation and activation in disease progression. Pharmacological inhibition of CSF1R in mice upregulated antiinflammatory mediators, including IL-4, IL-5, IL-13, and CCL9, and increased synapse and spine densities while reducing proinflammatory cytokine levels (64). Although targeting CSF1R has therapeutic potential, caution should be taken, as CSF1R haploinsufficiency was linked to adult-onset leukoencephalopathy with axonal spheroids and pigmented glia (145). Additionally, CSF1R signaling in a selected neuron population, rather than in microglia, provides neuroprotective and survival signals in the neurodegeneration setting (146).

\section{TREM2}

Triggering receptor expressed on myeloid cells-2 (TREM2) is abundantly expressed on microglia. Allelic variants of TREM2 confer high risk for developing sporadic AD; therefore, research into TREM2's function has gained momentum. TREM2 has many ligands, including phospholipids and sulfatides, which are exposed on apoptotic or damaged cells, lipoprotein particles such as HDL and LDL, and APOE. TREM2 promotes phagocytosis of apoptotic neurons, proliferation, and survival of microglia, and has been implicated in dampening TLR-induced inflammatory responses (147-151). Additionally, TREM2 deficiency impairs microglial phagocytosis of plaques opsonized with anti-A $\beta$ antibodies, suggesting a critical role for TREM2 in the efficacy of antibody-based therapeutics (152). Stimulation of nuclear receptor signaling with the retinoid $\mathrm{X}$ receptor agonist bexarotene induces the TREM2 expression in microglia (153). TREM2 ${ }^{+}$microglia show increased expression of phagocytic markers, suggesting that these microglia are phagocytic. Given that APOE binds TREM2, one may speculate that APOE increases phagocytosis of $A \beta$ by enhancing TREM 2 signaling.

AD risk variants in TREM2 impair the cell surface transport and expression of TREM2; some variants either impair or markedly enhance the binding of TREM2 to phospholipids, suggesting that balanced TREM2 function in AD is important to counteract disease progression (147). TREM2 levels were elevated in the microglia of AD patients, particularly in microglia associated with plaques and neurofibrillary changes, and TREM2 levels correlated with markers of neurodegeneration (154), suggesting a pathogenic role for TREM2.

Studies on TREM2 deficiency in AD mice have produced conflicting results, with reports that $A \beta$ pathology could be either increased or decreased. TREM2 was upregulated in $\mathrm{CD} 45^{\mathrm{hi}} \mathrm{LyC}^{+}$ myeloid cells surrounding the amyloid plaques, but not in parenchymal P2Y12 ${ }^{+}$microglia (155). TREM2 deficiency eliminated plaque-associated $\mathrm{CD}_{4} 5^{\mathrm{hi}} \mathrm{LyC}^{+}$myeloid cells, leading to reduced inflammation and AD-like pathology in these mice (155); however, TREM2-expressing myeloid cells were enriched in microglia processes contacting amyloid deposits in AD mice, and TREM2 deletion resulted in less compact plaques and increased dystrophic neurites. Similarly, TREM2 silencing in P301S mice increased tau pathology and synapse loss and enhanced the proinflammatory cytokine levels in the brain (156). A recent report indicated that the opposing results from previous work might reflect a disease progression-dependent influence of TREM2 deficiency on AD pathology. While TREM2 deficiency in young APP/PS1-21 mice ameliorated amyloid pathology, it enhanced amyloid deposition at later disease stages (157). Additionally, TREM2 deficiency in aged mice decreased plaque-associated myeloid cell accumulation by reducing proliferation (157). Considering the dual role of TREM2, a longitudinal assessment of TREM2 in conditional knockout models will reveal important effects of TREM2 signaling on myeloid cells during $\mathrm{AD}$ progression to support development of TREM2-directed therapeutics for different disease stages.

\section{Optogenics in activation of microglia}

Gamma oscillations arise from the activation of local circuits of excitatory and fast-spiking inhibitory neurons resonating at 20-50 $\mathrm{Hz}$, and changes in gamma oscillation have been observed in neurological diseases (158). Interestingly, entraining gamma oscillation at $40 \mathrm{~Hz}$ in $5 \mathrm{xFAD}$ mice induced amyloid reductions that were associated with an increase in microglial numbers and morphological changes such as soma enlargement and shortening of processes loaded with $A \beta$. RNA sequencing analysis showed an increase in phagocytic genes, such as $C d 68, \beta_{2}$-microglobulin $(B 2 m)$, Icam, and lysozyme 2 (Lyz2), and in the proliferation markers Csf1, Csf1r, and Csf2ra. Notably, levels of proinflammatory genes (Illb, Il6, $C d 11 b)$ and antiinflammatory genes (Igf1) were not changed (158), suggesting that it is possible to use immunomodulation to polarize microglia toward a beneficial activation state that is characterized 
by increased phagocytic activity without the induction of sustained inflammatory responses. Further studies are needed to evaluate the therapeutic potential of gamma oscillation in human AD.

\section{Concluding remarks}

Although the data are not fully consistent, they strongly suggest that microglia could be mobilized to promote $A \beta$ clearance and reduce amyloid deposition through multiple mechanisms. Given that age has a dramatic impact on innate immunity and represents a major risk factor for $\mathrm{AD}$, more longitudinal studies need to be performed and the overall outcome of immune modulation needs to be tested in aged animals. A plethora of evidence supports the dual nature of neuroinflammation in the early versus the late phases of AD. Additionally, the M1 or M2 microglia phenotypes could have a detrimental or beneficial outcome depending on the disease context, and more detailed analysis is required. Additionally, in vivo human studies, informed by animal models, will help better characterize the role of microglia activation in the dynamic stages of the disease. Interestingly, microglia seem to be intimately involved in the propagation of tau pathology; however, it remains unclear whether microglia contribute to tau pathology by failing to phagocytize pathological tau or by releasing factors that exacerbate tau pathology. From a therapeutic perspective, it will be interesting to identify the role of disease-specific microglial phenotypes during the progression of AD to design immunotherapy that boosts or tempers inflammation depending on the precise disease stage. One limitation of current mouse models is the absence of clear neurodegeneration and human-like tau pathology unless the disease is induced by a tau mutation. It seems that combining human induced pluripotent stem cell (iPSC) technology (159) with recent advances in gene editing will provide new opportunities to model disease pathology that more closely resembles human $\mathrm{AD}$.

\section{Acknowledgment}

This work has been supported by an ERA-NET consortium grant (Neuron-Webund Tracinflamm: O1EW1508) to MTH.

Address correspondence to: Michael T. Heneka, Department of Neurodegenerative Diseases and Gerontopsychiatry, Sigmund-Freud Strasse 25, 53127 Bonn, Germany. Phone: 49.228.28713092; Email: michael.heneka@ukbonn.de.
1. Beers DR, et al. Wild-type microglia extend survival in PU.1 knockout mice with familial amyotrophic lateral sclerosis. Proc Natl Acad Sci U S A. 2006;103(43):16021-16026.

2. Kierdorf K, et al. Microglia emerge from erythromyeloid precursors via Pu.1- and Irf8-dependent pathways. Nat Neurosci. 2013;16(3):273-280.

3. McKercher SR, et al. Targeted disruption of the PU.1 gene results in multiple hematopoietic abnormalities. EMBO J. 1996;15(20):5647-5658.

4. Schulz C, et al. A lineage of myeloid cells independent of Myb and hematopoietic stem cells. Science. 2012;336(6077):86-90.

5. Alliot F, Godin I, Pessac B. Microglia derive from progenitors, originating from the yolk sac, and which proliferate in the brain. Brain Res Dev Brain Res. 1999;117(2):145-152.

6. Ginhoux F, et al. Fate mapping analysis reveals that adult microglia derive from primitive macrophages. Science. 2010;330(6005):841-845.

7. Askew K, et al. Coupled Proliferation and Apoptosis Maintain the Rapid Turnover of Microglia in the Adult Brain. Cell Rep. 2017;18(2):391-405.

8. Ajami B, Bennett JL, Krieger C, Tetzlaff W, Rossi FM. Local self-renewal can sustain CNS microglia maintenance and function throughout adult life. Nat Neurosci. 2007;10(12):1538-1543.

9. Pierre WC, Smith PL, Londono I, Chemtob S, Mallard C, Lodygensky GA. Neonatal microglia: The cornerstone of brain fate. Brain Behav Immun. 2017;59:333-345.

10. Gomez-Nicola D, Perry VH. Microglial dynamics and role in the healthy and diseased brain: a paradigm of functional plasticity. Neuroscientist. 2015;21(2):169-184.

11. Lawson LJ, Perry VH, Dri P, Gordon S. Heterogeneity in the distribution and morphology of microglia in the normal adult mouse brain. Neuroscience. 1990;39(1):151-170.

12. Mittelbronn M, Dietz K, Schluesener HJ, Meyermann R. Local distribution of microglia in the normal adult human central nervous system differs by up to one order of magnitude. Acta Neuropathol. 2001;101(3):249-255.

13. Grabert K, et al. Microglial brain region-dependent diversity and selective regional sensitivities to aging. Nat Neurosci. 2016;19(3):504-516.

14. Parkhurst $\mathrm{CN}$, et al. Microglia promote learning-dependent synapse formation through brain-derived neurotrophic factor. Cell. 2013;155(7):1596-1609.

15. Udeochu JC, Shea JM, Villeda SA. Microglia communication: Parallels between aging and Alzheimer's disease. Clin Exp Neuroimmunol. 2016;7(2):114-125.

16. Nimmerjahn A, Kirchhoff F, Helmchen F. Resting microglial cells are highly dynamic surveillants of brain parenchyma in vivo. Science. 2005;308(5726):1314-1318.

17. Tremblay MÈ, Stevens B, Sierra A, Wake $\mathrm{H}$, Bessis A, Nimmerjahn A. The role of microglia in the healthy brain. J Neurosci. 2011;31(45):16064-16069.

18. Davalos D, et al. ATP mediates rapid microglial response to local brain injury in vivo. Nat Neurosci. 2005;8(6):752-758.

19. Haynes SE, et al. The P2Y12 receptor regulates microglial activation by extracellular nucleotides. Nat Neurosci. 2006;9(12):1512-1519.

20. Hristovska I, Pascual O. Deciphering resting microglial morphology and process motility from a synaptic prospect. Front Integr Neurosci. 2015;9:73.

21. Paolicelli RC, et al. Synaptic pruning by microglia is necessary for normal brain development. Science. 2011;333(6048):1456-1458.

22. Sierra A, et al. Microglia shape adult hippocampal neurogenesis through apoptosis-coupled phagocytosis. Cell Stem Cell. 2010;7(4):483-495.

23. Stevens B, et al. The classical complement cascade mediates CNS synapse elimination. Cell. 2007;131(6):1164-1178.

24. Tremblay MÈ, Lowery RL, Majewska AK.
Microglial interactions with synapses are modulated by visual experience. PLoS Biol. 2010;8(11):e1000527.

25. Wake H, Moorhouse AJ, Jinno S, Kohsaka S, Nabekura J. Resting microglia directly monitor the functional state of synapses in vivo and determine the fate of ischemic terminals. J Neurosci. 2009;29(13):3974-3980.

26. Gordon S. Alternative activation of macrophages. Nat Rev Immunol. 2003;3(1):23-35.

27. Varin A, Gordon S. Alternative activation of macrophages: immune function and cellular biology. Immunobiology. 2009;214(7):630-641.

28. Mantovani A, Sica A, Sozzani S, Allavena P, Vecchi A, Locati M. The chemokine system in diverse forms of macrophage activation and polarization. Trends Immunol. 2004;25(12):677-686.

29. Hume DA, Freeman TC. Transcriptomic analysis of mononuclear phagocyte differentiation and activation. Immunol Rev. 2014;262(1):74-84.

30. Xue J, et al. Transcriptome-based network analysis reveals a spectrum model of human macrophage activation. Immunity. 2014;40(2):274-288.

31. Colton CA. Heterogeneity of microglial activation in the innate immune response in the brain. J Neuroimmune Pharmacol. 2009;4(4):399-418.

32. Colonna M, Butovsky O. Microglia function in the central nervous system during health and neurodegeneration. Annu Rev Immunol. 2017;35:441-468.

33. Chiu IM, et al. A neurodegeneration-specific gene-expression signature of acutely isolated microglia from an amyotrophic lateral sclerosis mouse model. Cell Rep. 2013;4(2):385-401.

34. Holtman IR, et al. Induction of a common microglia gene expression signature by aging and neurodegenerative conditions: a co-expression metaanalysis. Acta Neuropathol Commun. 2015;3:31.

35. Wes PD, Holtman IR, Boddeke EW, Möller T, Eggen BJ. Next generation transcriptomics and genomics elucidate biological complexity of microglia in health and disease. Glia. 
2016;64(2):197-213.

36. Ransohoff RM. A polarizing question: do M1 and M2 microglia exist? Nat Neurosci. 2016;19(8):987-991.

37. Walker DG, Lue LF. Immune phenotypes of microglia in human neurodegenerative disease: challenges to detecting microglial polarization in human brains. Alzheimers Res Ther. 2015;7(1):56.

38. Bennett ML, et al. New tools for studying microglia in the mouse and human CNS. Proc Natl Acad Sci U S A. 2016;113(12):E1738-E1746.

39. Zhang Y, et al. An RNA-sequencing transcriptome and splicing database of glia, neurons, and vascular cells of the cerebral cortex. JNeurosci. 2014;34(36):11929-11947.

40. Alzheimer's Association. 2016 Alzheimer's disease facts and figures. Alzheimers Dement. 2016;12(4):459-509.

41. Goate A, et al. Segregation of a missense mutation in the amyloid precursor protein gene with familial Alzheimer's disease. Nature. 1991;349(6311):704-706.

42. Rogaev EI, et al. Familial Alzheimer's disease in kindreds with missense mutations in a gene on chromosome 1 related to the Alzheimer's disease type 3 gene. Nature. 1995;376(6543):775-778.

43. Sherrington R, et al. Cloning of a gene bearing missense mutations in early-onset familial Alzheimer's disease. Nature. 1995;375(6534):754-760.

44. Raber J, Huang Y, Ashford JW. ApoE genotype accounts for the vast majority of $\mathrm{AD}$ risk and $\mathrm{AD}$ pathology. Neurobiol Aging. 2004;25(5):641-650.

45. Terwel D, et al. Critical role of astroglial apolipoprotein $\mathrm{E}$ and liver $\mathrm{X}$ receptor- $\alpha$ expression for microglial A $\beta$ phagocytosis. J Neurosci. 2011;31(19):7049-7059.

46. Hyman BT, Marzloff K, Arriagada PV. The lack of accumulation of senile plaques or amyloid burden in Alzheimer's disease suggests a dynamic balance between amyloid deposition and resolution. J Neuropathol Exp Neurol. 1993;52(6):594-600.

47. Mawuenyega KG, et al. Decreased clearance of CNS beta-amyloid in Alzheimer's disease. Science. 2010;330(6012):1774

48. Heneka MT. Inflammasome activation and innate immunity in Alzheimer's disease. Brain Pathol. 2017;27(2):220-222.

49. Miners JS, Barua N, Kehoe PG, Gill S, Love S. $\mathrm{A} \beta$-degrading enzymes: potential for treatment of Alzheimer disease. J Neuropathol Exp Neurol. 2011;70(11):944-959.

50. Bakota L, Brandt R. Tau biology and taudirected therapies for Alzheimer's disease. Drugs. 2016;76(3):301-313.

51. Gautam V, D'Avanzo C, Berezovska O, Tanzi RE, Kovacs DM. Synaptotagmins interact with APP and promote $\mathrm{A} \beta$ generation. Mol Neurodegener. 2015;10:31.

52. Hardy J, Selkoe DJ. The amyloid hypothesis of Alzheimer's disease: progress and problems on the road to therapeutics. Science. 2002;297(5580):353-356.

53. Penazzi L, et al. A $\beta$-mediated spine changes in the hippocampus are microtubule-dependent and can be reversed by a subnanomolar concentration of the microtubule-stabilizing agent epothilone $\mathrm{D}$. Neuropharmacology. 2016;105:84-95.

54. Huang YA, Zhou B, Wernig M, Südhof TC.
ApoE2, ApoE3, and ApoE4 differentially stimulate APP transcription and A $\beta$ secretion. Cell. 2017;168(3):427-441.e21.

55. Lambert JC, et al. Meta-analysis of 74,046 individuals identifies 11 new susceptibility loci for Alzheimer's disease. Nat Genet. 2013;45(12):1452-1458.

56. Zhang B, et al. Integrated systems approach identifies genetic nodes and networks in late-onset Alzheimer's disease. Cell. 2013;153(3):707-720.

57. Heneka MT, Golenbock DT, Latz E. Innate immunity in Alzheimer's disease. Nat Immunol. 2015;16(3):229-236.

58. Eikelenboom P, Stam FC. Immunoglobulins and complement factors in senile plaques. An immunoperoxidase study. Acta Neuropathol. 1982;57(2-3):239-242.

59. Frautschy SA, et al. Microglial response to amyloid plaques in APPsw transgenic mice. Am J Pathol. 1998;152(1):307-317.

60. Griffin WS, et al. Brain interleukin 1 and S-100 immunoreactivity are elevated in Down syndrome and Alzheimer disease. Proc Natl Acad Sci U S A. 1989;86(19):7611-7615.

61. McGeer PL, Itagaki S, Tago H, McGeer EG. Reactive microglia in patients with senile dementia of the Alzheimer type are positive for the histocompatibility glycoprotein HLA-DR. Neurosci Lett. 1987;79(1-2):195-200.

62. Tooyama I, Kimura H, Akiyama H, McGeer PL. Reactive microglia express class I and class II major histocompatibility complex antigens in Alzheimer's disease. Brain Res. 1990;523(2):273-280.

63. Kamphuis W, Orre M, Kooijman L, Dahmen M, Hol EM. Differential cell proliferation in the cortex of the APPswePS1dE9 Alzheimer's disease mouse model. Glia. 2012;60(4):615-629.

64. Olmos-Alonso A, et al. Pharmacological targeting of CSF1R inhibits microglial proliferation and prevents the progression of Alzheimer's-like pathology. Brain. 2016;139(pt 3):891-907.

65. Kamphuis W, Kooijman L, Schetters S, Orre M, Hol EM. Transcriptional profiling of CD11c-positive microglia accumulating around amyloid plaques in a mouse model for Alzheimer's disease. Biochim Biophys Acta. 2016;1862(10):1847-1860.

66. Martin E, Boucher C, Fontaine B, Delarasse C. Distinct inflammatory phenotypes of microglia and monocyte-derived macrophages in Alzheimer's disease models: effects of aging and amyloid pathology. Aging Cell. 2017;16(1):27-38.

67. Bisht K, et al. Dark microglia: A new phenotype predominantly associated with pathological states. Glia. 2016;64(5):826-839.

68. Asai H, et al. Depletion of microglia and inhibition of exosome synthesis halt tau propagation. Nat Neurosci. 2015;18(11):1584-1593.

69. Liddelow SA, et al. Neurotoxic reactive astrocytes are induced by activated microglia. Nature. 2017;541(7638):481-487.

70. Streit WJ, Xue QS, Tischer J, Bechmann I. Microglial pathology. Acta Neuropathol Commun. 2014;2:142.

71. Venegas C, Heneka MT. Danger-associated molecular patterns in Alzheimer's disease. J Leukoc Biol. 2017;101(1):87-98.

72. Yang T, Li S, Xu H, Walsh DM, Selkoe DJ. Large soluble oligomers of amyloid $\beta$-protein from alzheimer brain are far less neuroactive than the smaller oligomers to which they dissociate. J Neurosci. 2017;37(1):152-163.

73. Yan SD, et al. RAGE and amyloid- $\beta$ peptide neurotoxicity in Alzheimer's disease. Nature. 1996;382(6593):685-691.

74. Landreth GE, Reed-Geaghan EG. Toll-like receptors in Alzheimer's disease. Curr Top Microbiol Immunol. 2009;336:137-153.

75. El Khoury J, Hickman SE, Thomas CA, Cao L, Silverstein SC, Loike JD. Scavenger receptormediated adhesion of microglia to beta-amyloid fibrils. Nature. 1996;382(6593):716-719.

76. Paresce DM, Ghosh RN, Maxfield FR. Microglial cells internalize aggregates of the Alzheimer's disease amyloid beta-protein via a scavenger receptor. Neuron. 1996;17(3):553-565.

77. Del Bo R, Angeretti N, Lucca E, De Simoni MG, Forloni G. Reciprocal control of inflammatory cytokines, IL-1 and IL-6, and beta-amyloid production in cultures. Neurosci Lett. 1995;188(1):70-74.

78. Pan XD, et al. Microglial phagocytosis induced by fibrillar $\beta$-amyloid is attenuated by oligomeric $\beta$-amyloid: implications for Alzheimer's disease. Mol Neurodegener. 2011;6:45.

79. Babcock AA, et al. Cytokine-producing microglia have an altered beta-amyloid load in aged APP/ PS1 Tg mice. Brain Behav Immun. 2015;48:86-101.

80. Taupenot L, Ciesielski-Treska J, Ulrich G, Chasserot-Golaz S, Aunis D, Bader MF. Chromogranin A triggers a phenotypic transformation and the generation of nitric oxide in brain microglial cells. Neuroscience. 1996;72(2):377-389.

81. Kummer MP, et al. Mrp14 deficiency ameliorates amyloid $\beta$ burden by increasing microglial phagocytosis and modulation of amyloid precursor protein processing. JNeurosci. 2012;32(49):17824-17829.

82. Schmid CD, et al. Differential gene expression in LPS/IFNgamma activated microglia and macrophages: in vitro versus in vivo. J Neurochem. 2009;109(suppl 1):117-125.

83. Junker A, et al. MicroRNA profiling of multiple sclerosis lesions identifies modulators of the regulatory protein CD47. Brain. 2009;132(pt 12):3342-3352.

84. Ransohoff RM, Cardona AE. The myeloid cells of the central nervous system parenchyma. Nature. 2010;468(7321):253-262.

85. Barclay AN, Wright GJ, Brooke G, Brown MH. CD200 and membrane protein interactions in the control of myeloid cells. Trends Immunol. 2002;23(6):285-290.

86. Hoek RM, et al. Down-regulation of the macrophage lineage through interaction with OX2 (CD200). Science. 2000;290(5497):1768-1771.

87. Harrison JK, et al. Role for neuronally derived fractalkine in mediating interactions between neurons and CX3CR1-expressing microglia. Proc Natl Acad Sci U S A. 1998;95(18):10896-10901.

88. Hundhausen C, et al. The disintegrin-like metalloproteinase ADAM10 is involved in constitutive cleavage of CX3CL1 (fractalkine) and regulates CX3CL1-mediated cell-cell adhesion. Blood. 2003;102(4):1186-1195

89. Mott RT, et al. Neuronal expression of CD22: novel mechanism for inhibiting microglial proinflammatory cytokine production. Glia. 2004;46(4):369-379. 
90. Smith RE, et al. A novel MyD-1 (SIRP-1alpha) signaling pathway that inhibits LPS-induced TNF- $\alpha$ production by monocytes. Blood. 2003;102(7):2532-2540.

91. Gahmberg CG, Tian L, Ning L, Nyman-Huttunen H. ICAM-5 - a novel two-facetted adhesion molecule in the mammalian brain. Immunol Lett. 2008;117(2):131-135.

92. Sheridan GK, Murphy KJ. Neuron-glia crosstalk in health and disease: fractalkine and CX3CR1 take centre stage. Open Biol. 2013;3(12):130181

93. El Khoury J, et al. Ccr2 deficiency impairs microglial accumulation and accelerates progression of Alzheimer-like disease. Nat Med. 2007;13(4):432-438.

94. Fuhrmann M, et al. Microglial Cx3cr1 knockout prevents neuron loss in a mouse model of Alzheimer's disease. Nat Neurosci. 2010;13(4):411-413.

95. Krauthausen M, et al. CXCR3 promotes plaque formation and behavioral deficits in an Alzheimer's disease model. JClin Invest. 2015;125(1):365-378.

96. Lee $S$, et al. CX3CR1 deficiency alters microglial activation and reduces $\beta$-amyloid deposition in two Alzheimer's disease mouse models. Am J Pathol. 2010;177(5):2549-2562.

97. Lee S, et al. Opposing effects of membraneanchored CX3CL1 on amyloid and tau pathologies via the p38 MAPK pathway. J Neurosci. 2014;34(37):12538-12546.

98. Heneka MT, et al. Locus ceruleus controls Alzheimer's disease pathology by modulating microglial functions through norepinephrine. Proc Natl Acad SciU S A. 2010;107(13):6058-6063.

99. Chalermpalanupap T, et al. Targeting norepinephrine in mild cognitive impairment and Alzheimer's disease. Alzheimers Res Ther. 2013;5(2):21.

100.Hamelin L, et al. Early and protective microglial activation in Alzheimer's disease: a prospective study using 18F-DPA-714 PET imaging. Brain. 2016;139(pt 4):1252-1264.

101.Brosseron F, Krauthausen M, Kummer M, Heneka MT. Body fluid cytokine levels in mild cognitive impairment and Alzheimer's disease: a comparative overview. Mol Neurobiol. 2014;50(2):534-544.

102. Fan Z, Brooks DJ, Okello A, Edison P. An early and late peak in microglial activation in Alzheimer's disease trajectory. Brain. 2017;140(3):792-803.

103. Fan Z, Okello AA, Brooks DJ, Edison P. Longitudinal influence of microglial activation and amyloid on neuronal function in Alzheimer's disease. Brain. 2015;138(pt 12):3685-3698.

104.Leoutsakos JM, Muthen BO, Breitner JC, Lyketsos CG, ADAPT Research Team. Effects of non-steroidal anti-inflammatory drug treatments on cognitive decline vary by phase of pre-clinical Alzheimer disease: findings from the randomized controlled Alzheimer's Disease Antiinflammatory Prevention Trial. Int J Geriatr Psychiatry. 2012;27(4):364-374.

105. Hoozemans JJ, Rozemuller JM, van Haastert ES, Veerhuis R, Eikelenboom P. Cyclooxygenase- 1 and -2 in the different stages of Alzheimer's disease pathology. Curr Pharm Des. 2008;14(14):1419-1427.

106. Hoozemans JJ, Rozemuller AJ, van Haastert ES, Eikelenboom P, van Gool WA. Neuroinflamma- tion in Alzheimer's disease wanes with age. J Neuroinflammation. 2011;8:171.

107. Frank S, Copanaki E, Burbach GJ, Müller UC, Deller T. Differential regulation of toll-like receptor mRNAs in amyloid plaque-associated brain tissue of aged APP23 transgenic mice. Neurosci Lett. 2009;453(1):41-44.

108. Stewart CR, et al. CD36 ligands promote sterile inflammation through assembly of a Toll-like receptor 4 and 6 heterodimer. Nat Immunol. 2010;11(2):155-161.

109. Lee DC, Rizer J, Hunt JB, Selenica ML, Gordon MN, Morgan D. Review: experimental manipulations of microglia in mouse models of Alzheimer's pathology: activation reduces amyloid but hastens tau pathology. Neuropathol Appl Neurobiol. 2013;39(1):69-85.

110.Song $\mathrm{M}$, et al. TLR4 mutation reduces microglial activation, increases $A \beta$ deposits and exacerbates cognitive deficits in a mouse model of Alzheimer's disease. J Neuroinflammation. 2011;8:92.

111. Herber DL, Maloney JL, Roth LM, Freeman MJ, Morgan D, Gordon MN. Diverse microglial responses after intrahippocampal administration of lipopolysaccharide. Glia. 2006;53(4):382-391.

112. Qiao X, Cummins DJ, Paul SM. Neuroinflammation-induced acceleration of amyloid deposition in the APPV717F transgenic mouse. Eur J Neurosci. 2001;14(3):474-482.

113. Sheng JG, Bora SH, Xu G, Borchelt DR, Price DL, Koliatsos VE. Lipopolysaccharide-inducedneuroinflammation increases intracellular accumulation of amyloid precursor protein and amyloid beta peptide in APPswe transgenic mice. Neurobiol Dis. 2003;14(1):133-145

114. Reed-Geaghan EG, Reed QW, Cramer PE, Landreth GE. Deletion of CD14 attenuates Alzheimer's disease pathology by influencing the brain's inflammatory milieu. J Neurosci. 2010;30(46):15369-15373.

115. McDonald CL, et al. Inhibiting TLR2 activation attenuates amyloid accumulation and glial activation in a mouse model of Alzheimer's disease. Brain Behav Immun. 2016;58:191-200.

116. Scholtzova $\mathrm{H}$, et al. Innate immunity stimulation via toll-like receptor 9 ameliorates vascular amyloid pathology in Tg-SwDI mice with associated cognitive benefits. J Neurosci. 2017;37(4):936-959.

117. Scholtzova H, et al. Amyloid $\beta$ and Tau Alzheimer's disease related pathology is reduced by Toll-like receptor 9 stimulation. Acta Neuropathol Commun. 2014;2:101

118. Doi Y, et al. Microglia activated with the toll-like receptor 9 ligand $\mathrm{CpG}$ attenuate oligomeric amyloid \{beta\} neurotoxicity in in vitro and in vivo models of Alzheimer's disease. Am J Pathol. 2009;175(5):2121-2132.

119. Wang YL, et al. Toll-like receptor 9 promoter polymorphism is associated with decreased risk of Alzheimer's disease in Han Chinese. J Neuroinflammation. 2013;10:101.

120. Crehan H, Holton P, Wray S, Pocock J, Guerreiro R, Hardy J. Complement receptor 1 (CR1) and Alzheimer's disease. Immunobiology. 2012;217(2):244-250.

121. Hong S, et al. Complement and microglia mediate early synapse loss in Alzheimer mouse models. Science. 2016;352(6286):712-716.
122. Wyss-Coray T, et al. Prominent neurodegeneration and increased plaque formation in complementinhibited Alzheimer's mice. Proc Natl Acad Sci US A. 2002;99(16):10837-10842.

123. Maier M, Peng Y, Jiang L, Seabrook TJ, Carroll MC, Lemere CA. Complement C3 deficiency leads to accelerated amyloid beta plaque deposition and neurodegeneration and modulation of the microglia/macrophage phenotype in amyloid precursor protein transgenic mice. J Neurosci. 2008;28(25):6333-6341.

124. Fonseca MI, et al. Treatment with a C5aR antagonist decreases pathology and enhances behavioral performance in murine models of Alzheimer's disease. JImmunol. 2009;183(2):1375-1383.

125. Lian H, Litvinchuk A, Chiang AC, Aithmitti N, Jankowsky JL, Zheng H. Astrocyte-microglia cross talk through complement activation modulates amyloid pathology in mouse models of Alzheimer's disease. J Neurosci. 2016;36(2):577-589.

126.Griffin WS, Mrak RE. Interleukin-1 in the genesis and progression of and risk for development of neuronal degeneration in Alzheimer's disease. JLeukoc Biol. 2002;72(2):233-238.

127. Chakrabarty $P$, et al. Massive gliosis induced by interleukin- 6 suppresses $A \beta$ deposition in vivo: evidence against inflammation as a driving force for amyloid deposition. FASEB $J$. 2010;24(2):548-559.

128. Chakrabarty P, et al. IFN- $\gamma$ promotes complement expression and attenuates amyloid plaque deposition in amyloid beta precursor protein transgenic mice. J Immunol. 2010;184(9):5333-5343.

129. Montgomery SL, et al. Ablation of TNF-RI/RII expression in Alzheimer's disease mice leads to an unexpected enhancement of pathology: implications for chronic pan-TNF- $\alpha$ suppressive therapeutic strategies in the brain. Am J Pathol. 2011;179(4):2053-2070.

130. Vom Berg J, et al. Inhibition of IL-12/IL-23 signaling reduces Alzheimer's disease-like pathology and cognitive decline. Nat Med. 2012;18(12):1812-1819.

131. Heneka MT, et al. NLRP3 is activated in Alzheimer's disease and contributes to pathology in APP/PS1 mice. Nature. 2013;493(7434):674-678.

132. Chakrabarty P, et al. IL-10 alters immunoproteostasis in APP mice, increasing plaque burden and worsening cognitive behavior. Neuron . 2015;85(3):519-533.

133. Guillot-Sestier MV, et al. Il10 deficiency rebalances innate immunity to mitigate Alzheimerlike pathology. Neuron. 2015;85(3):534-548.

134. Gehrmann J, Banati RB. Microglial turnover in the injured CNS: activated microglia undergo delayed DNA fragmentation following peripheral nerve injury. J Neuropathol Exp Neurol. 1995;54(5):680-688.

135. Gómez-Nicola D, Fransen NL, Suzzi S, Perry VH. Regulation of microglial proliferation during chronic neurodegeneration. J Neurosci. 2013;33(6):2481-2493.

136. Doorn KJ, Drukarch B, van Dam AM, Lucassen PJ. Hippocampal proliferation is increased in presymptomatic Parkinson's disease and due to microglia. Neural Plast. 2014;2014:959154.

137. Boekhoorn K, Joels M, Lucassen PJ. Increased proliferation reflects glial and vascular- 
associated changes, but not neurogenesis in the presenile Alzheimer hippocampus. Neurobiol Dis. 2006;24(1):1-14.

138. Lalancette-Hébert M, Gowing G, Simard A, Weng YC, Kriz J. Selective ablation of proliferating microglial cells exacerbates ischemic injury in the brain. J Neurosci. 2007;27(10):2596-2605.

139. Denes A, et al. Proliferating resident microglia after focal cerebral ischaemia in mice. JCereb Blood Flow Metab. 2007;27(12):1941-1953.

140.Ehninger D, Kempermann G. Regional effects of wheel running and environmental enrichment on cell genesis and microglia proliferation in the adult murine neocortex. Cereb Cortex. 2003;13(8):845-851.

141. Lawson LJ, Perry VH, Gordon S. Turnover of resident microglia in the normal adult mouse brain. Neuroscience. 1992;48(2):405-415.

142. Ulland TK, Wang Y, Colonna M. Regulation of microglial survival and proliferation in health and diseases. Semin Immunol. 2015;27(6):410-415.

143. Friedman AD. Transcriptional control of granulocyte and monocyte development. Oncogene. 2007;26(47):6816-6828.

144. Manz MG, Boettcher S. Emergency granulopoiesis. Nat Rev Immunol. 2014;14(5):302-314.

145. Chitu V, et al. Phenotypic characterization of a Csflr haploinsufficient mouse model of adult-onset leukodystrophy with axonal spheroids and pigmented glia (ALSP). Neurobiol Dis. 2015;74:219-228.

146.Luo J, et al. Colony-stimulating factor 1 receptor (CSF1R) signaling in injured neurons facilitates protection and survival. J Exp Med. 2013;210(1):157-172.

147. Colonna M, Butovsky O. Microglia function in the central nervous system during health and neurodegeneration. Annu Rev Immunol. 2017;35:441-468.

148. Hamerman JA, Jarjoura JR, Humphrey MB, Nakamura MC, Seaman WE, Lanier LL. Cutting edge: inhibition of TLR and FCR responses in macrophages by triggering receptor expressed on myeloid cells (TREM)-2 and DAP12.J Immunol. 2006;177(4):2051-2055.

149. Hsieh CL, et al. A role for TREM2 ligands in the phagocytosis of apoptotic neuronal cells by microglia. J Neurochem. 2009;109(4):1144-1156.

150. Takahashi K, Rochford CD, Neumann H. Clearance of apoptotic neurons without inflammation by microglial triggering receptor expressed on myeloid cells-2.J Exp Med. 2005;201(4):647-657.

151. Zheng H, et al. TREM2 promotes microglial survival by activating $\mathrm{Wnt} / \beta$-catenin pathway. J Neurosci. 2017;37(7):1772-1784.

152. Xiang X, et al. TREM2 deficiency reduces the efficacy of immunotherapeutic amyloid clear- ance. EMBO Mol Med.2016;8(9):992-1004.

153. Savage JC, et al. Nuclear receptors license phagocytosis by trem 2+ myeloid cells in mouse models of Alzheimer's disease. J Neurosci. 2015;35(16):6532-6543.

154.Lue LF, Schmitz CT, Serrano G, Sue LI, Beach TG, Walker DG. TREM2 protein expression changes correlate with Alzheimer's disease neurodegenerative pathologies in post-mortem temporal cortices. Brain Pathol. 2015;25(4):469-480.

155. Jay TR, et al. TREM2 deficiency eliminates TREM2+ inflammatory macrophages and ameliorates pathology in Alzheimer's disease mouse models. J Exp Med. 2015;212(3):287-295.

156.Jiang T, et al. Silencing of TREM2 exacerbates tau pathology, neurodegenerative changes, and spatial learning deficits in P301S tau transgenic mice. Neurobiol Aging. 2015;36(12):3176-3186.

157. Jay TR, et al. Disease progression-dependent effects of TREM2 deficiency in a mouse model of alzheimer's disease. JNeurosci. 2017;37(3):637-647.

158. Iaccarino HF, et al. Gamma frequency entrainment attenuates amyloid load and modifies microglia. Nature. 2016;540(7632):230-235.

159. Espuny-Camacho I, et al. Hallmarks of Alzheimer's disease in stem-cell-derived human neurons transplanted into mouse brain. Neuron . 2017;93(5):1066-1081.e8. 